\title{
Flexoelectric Effect in Biaxial Nematics
}

\author{
A. KAPANOWSKI* \\ Marian Smoluchowski Institute of Physics, Jagiellonian University \\ W.S. Reymonta 4, 30-059 Cracow, Poland
}

(Received January 13, 2012; in final form March 23, 2012)

\begin{abstract}
The flexoelectric effect provides a linear coupling between electric polarization and orientational deformation in liquid crystals. It influences many electrooptical phenomena and it is used in some bistable nematic devices. A statistical theory of dipole flexoelectric polarization in biaxial nematic liquid crystals is used to calculate temperature dependence of order parameters, elastic constants, and flexoelectric coefficients. The splitting of the two Meyer flexoelectric coefficients and the appearance of new flexoelectric coefficients is obtained at the uniaxial-biaxial nematic transition. The ordering of the split flexoelectric coefficients corresponds to the ordering of the split elastic constants.
\end{abstract}

PACS: $61.30 . \mathrm{Cz}, 77.84 . \mathrm{Nh}$

\section{Introduction}

The biaxial nematic liquid crystals were first predicted by Freiser [1], who showed that molecules with shapes that deviate from cylindrical symmetry could possess a nematic phase with three distinct optical axes. A biaxial nematic phase was first observed in a lyotropic mixture by $\mathrm{Yu}$ and Saupe [2] in 1980 but the existence of a thermotropic biaxial system was not certain for many years. Several reports appeared in 2004 on thermotropic liquid crystals formed by side-chain polymers [3], bent-core molecules [4, 5], and tetrapodes [6]. The number of new biaxial systems is constantly growing.

Many theoretical papers [7] and computer simulations [8] show that the molecular shape and pair interaction biaxiality are important for the biaxial phase to exist. However, very often real systems favour packing in the smectic or crystalline biaxial phases. It is a challenge for the theory to find factors responsible for absolute stability of the biaxial nematic phase. It was shown that fluctuations in molecular shape can influence the biaxial nematic phase stability [9]. The motivation for this search ranges from purely academic interest to the potential usage of these materials in faster displays, where in principle the commutation of the secondary director should give lower response times compared to the conventional twisted nematic and ferroelectric smectic devices.

A static electric field imposed on a nematic liquid crystal have many physical effects, but the most important are two of them. One is connected with the anisotropy

\footnotetext{
* e-mail: andrzej.kapanowski@uj.edu.pl
}

of the dielectric constant. The second effect is the appearance of the spontaneous polarization in a deformed liquid crystal; this is called the flexoelectric (FE) effect. Conversely, an electric field may induce distortions in the bulk. In 1969 Meyer showed that it is a steric effect due to the shape asymmetry of polar molecules [10]. In case of nonpolar molecules the FE effect originates from a gradient of quadrupole moment density [11].

The two FE coefficients were introduced by Meyer for splay and bend distortions of the uniaxial nematic phase [10]. Recently, a statistical theory for the dipole FE polarization was derived in the case of the biaxial nematic phase composed of $C_{2 v}$ molecules [12]. There are six splay-bend deformations of the biaxial nematic phase and thus six FE coefficients are defined, but only five of them are independent. General microscopic expressions for the FE coefficients involve the one-particle distribution function and the potential energy of two-body short-ranged interactions.

The FE effect has a large influence on many phenomena in liquid crystals: electrooptical phenomena and defect formation, for instance. It plays a key role in some device applications. Flexoelectric switching is important in bistable displays [13-15]. Flexoelectric coupling in chiral and twisted nematic crystals [16] leads to a linear rotation of the optic axis and also leads to device applications [17]. This flexoelectric-optic effect can be studied in the uniform lying helix configuration or in the uniform standing helix configuration [18]. Flexoelectric coupling in smectic liquid crystals has been shown to stabilize helical structures [19]. The FE effect was shown to stabilise blue phases and strongly widen their temperature range [20]. 
The FE polarization appears when some nematic distortions break the symmetry of the phase. Let us note that another way of breaking the nematic symmetry is to vary in space the order parameter, for example in the vicinity of a boundary surface. In that case, the ordo-electric polarization appears [21]. Recently, the influence of the flexoelectricity and the order electricity on the stability of defect structures was studied in nematic droplets with planar and homeotropic surface anchoring [22].

The purpose of this study is to calculate the values of the FE coefficients and other material parameters for model systems. The proper form of the interaction potential energy allows us to calculate the temperature dependence of the order parameters, the FE coefficients, and the elastic constants. The uniaxial and biaxial nematic phases are considered.

\section{Description of the system}

Let us consider a set of $N$ molecules contained in a volume $V$, at the temperature $T$. The molecules are rigid blocks ( $C_{2 v}$ symmetry) with three translational and three rotational degrees of freedom. It is assumed that the molecules interact via two-body short-range forces that depend on the distance between the molecules $\left(\boldsymbol{u}=\boldsymbol{r}_{2}\right.$ $\left.\boldsymbol{r}_{1}=u \boldsymbol{\Delta}\right)$ and their orientations described by the three Euler angles $R=(\phi, \theta, \psi)$ or by the three orthonormal vectors $(\boldsymbol{l}, \boldsymbol{m}, \boldsymbol{n})$. by

The microscopic free energy $F$ for the system is given

$$
\begin{aligned}
& F=F_{\text {ent }}+F_{\text {int }}, \\
& \beta F_{\text {ent }}=\int \mathrm{d} \boldsymbol{r} \mathrm{d} R G(\boldsymbol{r}, R)[\ln (G(\boldsymbol{r}, R) \Lambda)-1], \\
& \beta F_{\text {int }}= \\
& -\frac{1}{2} \int \mathrm{d} \boldsymbol{r}_{1} \mathrm{~d} R_{1} \mathrm{~d} \boldsymbol{r}_{2} \mathrm{~d} R_{2} G\left(\boldsymbol{r}_{1}, R_{1}\right) G\left(\boldsymbol{r}_{2}, R_{2}\right) f_{12} .
\end{aligned}
$$

Here $f_{12}=\exp \left(-\beta \Phi_{12}\right)-1$ is the Mayer function, $\Phi_{12}-$ the potential energy of interactions, $\mathrm{d} R=\mathrm{d} \phi \mathrm{d} \theta \sin \theta \mathrm{d} \psi$, $\beta=1 /\left(k_{\mathrm{B}} T\right)$, and $\Lambda$ is related to the ideal gas properties. The one-particle distribution function $G$ has the normalization

$$
\int \mathrm{d} \boldsymbol{r} \mathrm{d} R G(\boldsymbol{r}, R)=N
$$

The equilibrium distribution $G$ minimizing the free energy (1) satisfies the equation

$$
\ln \left(G\left(\boldsymbol{r}_{1}, R_{1}\right) \Lambda\right)-\int \mathrm{d} \boldsymbol{r}_{2} \mathrm{~d} R_{2} G\left(\boldsymbol{r}_{2}, R_{2}\right) f_{12}=\text { const. }
$$

For the homogeneous phase $G=G_{0}$ does not depend on the position and it has the form [23]:

$$
G_{0}(R)=G_{0}(\boldsymbol{l} \cdot \boldsymbol{L}, \boldsymbol{l} \cdot \boldsymbol{N}, \boldsymbol{n} \cdot \boldsymbol{L}, \boldsymbol{n} \cdot \boldsymbol{N}),
$$

where the orthonormal vectors $(\boldsymbol{L}, \boldsymbol{M}, \boldsymbol{N})$ define the biaxial nematic phase axes. In practice we characterize the alignment not through the full function $G$, but by some numerical parameters - order parameters. In the case of the biaxial nematic phase the main order parameters are the orientational distribution averages of the following four functions [24]: $F_{00}^{(2)}, F_{02}^{(2)}, F_{20}^{(2)}$, and $F_{22}^{(2)}$. We note that there are other notations [25]. In the uniaxial nematic phase the functions $F_{00}^{(2)}$ and $F_{02}^{(2)}$ have nonzero averages only. The molecule alignment can be also described by order tensors $Q$ which are often calculated for computer simulations [26] where the molecular and laboratory axes must be distinguished. The order tensors are defined as

$$
\begin{aligned}
& Q_{\alpha \beta}^{l l}=\left(3 l_{\alpha} l_{\beta}-\delta_{\alpha \beta}\right) / 2, \\
& Q_{\alpha \beta}^{m m}=\left(3 m_{\alpha} m_{\beta}-\delta_{\alpha \beta}\right) / 2, \\
& Q_{\alpha \beta}^{n n}=\left(3 n_{\alpha} n_{\beta}-\delta_{\alpha \beta}\right) / 2 .
\end{aligned}
$$

In our calculations we use diagonal values of the tensors $Q_{\alpha \beta}^{l l}$ and $Q_{\alpha \beta}^{n n}$. In the case of the perfect uniaxial nematic phase composed of the rod-like molecules and oriented along the $Z$ axis, we get $Q_{x x}^{n n}=Q_{y y}^{n n}=-1 / 2, Q_{z z}^{n n}=1$, $Q_{x x}^{l l}=Q_{y y}^{l l}=1 / 4$, and $Q_{z z}^{l l}=-1 / 2$. Let us note that $Q_{x x}^{l l}$ and $Q_{y y}^{l l}$ are positive. In the case of the perfect biaxial nematic phase we have $Q_{x x}^{l l}=Q_{y y}^{m m}=Q_{z z}^{n n}=1$.

\section{Elastic deformations of the phase}

Orientational ordering of biaxial nematics is usually described by the three orthonormal vectors

$$
\boldsymbol{L}=R_{1 \alpha} \boldsymbol{e}_{\alpha}, \quad \boldsymbol{M}=R_{2 \alpha} \boldsymbol{e}_{\alpha}, \quad \boldsymbol{N}=R_{3 \alpha} \boldsymbol{e}_{\alpha},
$$

where $\left(\boldsymbol{e}_{x}, \boldsymbol{e}_{y}, \boldsymbol{e}_{z}\right)$ is a space-fixed reference frame. The matrix elements $R_{i \alpha}(i=1,2,3$ and $\alpha=x, y, z)$ satisfy the conditions that express the orthogonality and the completeness of the local frame

$$
R_{i \alpha} R_{j \alpha}=\delta_{i j}, \quad R_{i \alpha} R_{i \beta}=\delta_{\alpha \beta} .
$$

In the homogeneous phase the vectors $(\boldsymbol{L}, \boldsymbol{M}, \boldsymbol{N})$ are constant in space, but in a deformed phase they depend on the position in space. In a continuum approach the distortion free-energy density $f_{\mathrm{d}}$ is obtained as an expansion about an undistorted reference state with respect to gradients of the vectors $(\boldsymbol{L}, \boldsymbol{M}, \boldsymbol{N})$. The form of the $f_{\mathrm{d}}$ can be derived in many alternative ways but we use the form presented by Stallinga and Vertogen [27] (the surface terms are neglected):

$$
\begin{aligned}
f_{\mathrm{d}} & =\frac{1}{2} K_{1111}\left(D_{11}\right)^{2}+\frac{1}{2} K_{1212}\left(D_{12}\right)^{2}+\frac{1}{2} K_{1313}\left(D_{13}\right)^{2} \\
& +\frac{1}{2} K_{2121}\left(D_{21}\right)^{2}+\frac{1}{2} K_{2222}\left(D_{22}\right)^{2}+\frac{1}{2} K_{2323}\left(D_{23}\right)^{2} \\
& +\frac{1}{2} K_{3131}\left(D_{31}\right)^{2}+\frac{1}{2} K_{3232}\left(D_{32}\right)^{2}+\frac{1}{2} K_{3333}\left(D_{33}\right)^{2} \\
& +K_{1122} D_{11} D_{22}+K_{1133} D_{11} D_{33}+K_{2233} D_{22} D_{33} \\
& +K_{1221} D_{12} D_{21}+K_{1331} D_{13} D_{31}+K_{2332} D_{23} D_{32} \\
D_{i j} & =\frac{1}{2} \epsilon_{j k l} R_{i \alpha} R_{k \beta} \partial_{\alpha} R_{l \beta} .
\end{aligned}
$$

Microscopic expressions for the elastic constants $K_{\mathrm{ijkl}}$ 
were derived in [23] and it was shown that there are 12 independent bulk constants because

$$
K_{1221}=K_{1122}, \quad K_{1331}=K_{1133}, \quad K_{2332}=K_{2233} .
$$

\section{Flexoelectric polarization}

Liquid crystalline phases often consist of polar molecules but in homogeneous nematic phases the average polarization is zero. On the other hand, a phase distortion can produce a polarization and this is called the FE effect. In a continuum approach the FE polarization of the biaxial nematic phase depends on the spatial derivatives of the vectors $(\boldsymbol{L}, \boldsymbol{M}, \boldsymbol{N})[12]$ :

$$
P_{\alpha}=\sum_{i}\left(s_{i i} R_{i \alpha} \partial_{\beta} R_{i \beta}+b_{i i} R_{i \beta} \partial_{\beta} R_{i \alpha}\right) .
$$

The parameters $s_{i i}$ and $b_{i i}(i=1,2,3)$ are not unique because if we add any constant to all of them, the polarization will not change. The physical FE coefficients $a_{i}$ $(i=4, \ldots, 9)$ are

$$
\begin{array}{lll}
a_{4}=s_{33}-b_{11}, & a_{5}=s_{22}-b_{11}, & a_{6}=s_{33}-b_{22}, \\
a_{7}=s_{11}-b_{22}, & a_{8}=s_{22}-b_{33}, & a_{9}=s_{11}-b_{33} .
\end{array}
$$

The coefficients satisfy the identity

$$
a_{4}-a_{5}-a_{6}+a_{7}+a_{8}-a_{9}=0 .
$$

Deformations of the biaxial nematic phase connected with the FE effect are given in Table I. In the case of the uniaxial nematic phase the FE polarization has the form

$$
P_{\alpha}=e_{1} N_{\alpha} \partial_{\beta} N_{\beta}+e_{3} N_{\beta} \partial_{\beta} N_{\alpha} .
$$

\section{TABLE I}

Deformations of the biaxial nematic phase connected with the FE effect. The corresponding elastic constants and the FE coefficients are given, the values for the uniaxial nematic phase are in parentheses.

\begin{tabular}{c|c|c}
\hline \hline Deformation & Elastic constant & FE coefficient \\
\hline $\boldsymbol{N}$ splay, $\boldsymbol{L}$ bend & $K_{1212}\left(K_{1}\right)$ & $a_{4}\left(e_{1}\right)$ \\
$\boldsymbol{M}$ splay, $\boldsymbol{L}$ bend & $K_{1313}(0)$ & $a_{5}(0)$ \\
$\boldsymbol{N}$ splay, $\boldsymbol{M}$ bend & $K_{2121}\left(K_{1}\right)$ & $a_{6}\left(e_{1}\right)$ \\
$\boldsymbol{L}$ splay, $\boldsymbol{M}$ bend & $K_{2323}(0)$ & $a_{7}(0)$ \\
$\boldsymbol{M}$ splay, $\boldsymbol{N}$ bend & $K_{3131}\left(K_{3}\right)$ & $a_{8}\left(-e_{3}\right)$ \\
$\boldsymbol{L}$ splay, $\boldsymbol{N}$ bend & $K_{3232}\left(K_{3}\right)$ & $a_{9}\left(-e_{3}\right)$
\end{tabular}

Let us define the molecule electric dipole moment as

$$
\mu_{\alpha}=\mu_{1} l_{\alpha}+\mu_{2} m_{\alpha}+\mu_{3} n_{\alpha} .
$$

In the case of the molecular iteractions described below, the FE coefficients can be expressed as follows:

$$
a_{4}=\int \mathrm{d} \boldsymbol{u} \mathrm{d} R_{1} \mathrm{~d} R_{2} f_{12} G_{0}\left(R_{1}\right) \mu_{3} n_{1 z}
$$

$$
\begin{aligned}
& \times\left(-u_{x}\right)\left(U_{2 z}-W_{2 x}\right), \\
a_{5} & =\int \mathrm{d} \boldsymbol{u} \mathrm{d} R_{1} \mathrm{~d} R_{2} f_{12} G_{0}\left(R_{1}\right) \mu_{3} n_{1 y}\left(-u_{x}\right) U_{2 y}, \\
a_{6} & =\int \mathrm{d} \boldsymbol{u} \mathrm{d} R_{1} \mathrm{~d} R_{2} f_{12} G_{0}\left(R_{1}\right) \mu_{3} n_{1 z} u_{y} W_{2 y}, \\
a_{7} & =\int \mathrm{d} \boldsymbol{u} \mathrm{d} R_{1} \mathrm{~d} R_{2} f_{12} G_{0}\left(R_{1}\right) \mu_{3} n_{1 x} u_{y} U_{2 y}, \\
a_{8} & =\int \mathrm{d} \boldsymbol{u} \mathrm{d} R_{1} \mathrm{~d} R_{2} f_{12} G_{0}\left(R_{1}\right) \mu_{3} n_{1 y}\left(-u_{z}\right) W_{2 y} \\
a_{9} & =\int \mathrm{d} \boldsymbol{u} \mathrm{d} R_{1} \mathrm{~d} R_{2} f_{12} G_{0}\left(R_{1}\right) \mu_{3} n_{1 x} u_{z}\left(U_{2 z}-W_{2 x}\right),
\end{aligned}
$$

where it is assumed that $\boldsymbol{n}$ defines the molecule $C_{2}$ axis and

$$
U_{\alpha}=\partial_{1} G_{0} l_{\alpha}+\partial_{3} G_{0} n_{\alpha}, \quad W_{\alpha}=\partial_{2} G_{0} l_{\alpha}+\partial_{4} G_{0} n_{\alpha} .
$$

\section{Results}

We performed our calculations for the square-well potential energy of the form

$$
\Phi_{12}(u / \sigma)= \begin{cases}+\infty & \text { for }(u / \sigma)<1, \\ -\epsilon & \text { for } 1<(u / \sigma)<2, \\ 0 & \text { for }(u / \sigma)>2,\end{cases}
$$

were $\epsilon$ is the depth of the well and $\sigma$ depends on the molecule orientations and on the vector $\Delta$ :

$$
\begin{aligned}
\sigma & =\sigma_{0}+\sigma_{1}\left(\boldsymbol{\Delta} \cdot \boldsymbol{n}_{1}-\boldsymbol{\Delta} \cdot \boldsymbol{n}_{2}\right) \\
& +\sigma_{2}\left[\left(\boldsymbol{\Delta} \cdot \boldsymbol{n}_{1}\right)^{2}+\left(\boldsymbol{\Delta} \cdot \boldsymbol{n}_{2}\right)^{2}\right] \\
& +\sigma_{3}\left[\left(\boldsymbol{\Delta} \cdot \boldsymbol{l}_{1}\right)^{2}+\left(\boldsymbol{\Delta} \cdot \boldsymbol{l}_{2}\right)^{2}\right] .
\end{aligned}
$$

The parameter $\sigma_{0}$ defines the length scale, $\sigma_{1}$ defines the FE term, $\sigma_{2}$ and $\sigma_{3}$ define biaxial nematic terms. We used the density $N V_{\mathrm{mol}} / V=0.1$, the molecule volume $V_{\text {mol }}$ was estimated from the mutually excluded volume. The FE coefficients were expressed in $\mu_{i} / \sigma_{0}^{2}$, the elastic constants in $\epsilon / \sigma_{0}$, and the temperature in $\epsilon / k_{\mathrm{B}}$. The parameters $\sigma_{i}$ are given in Table II. The two physical systems are considered that consist of wedge-shaped and banana-shaped molecules.

Parameters $\sigma_{i}$ used in calculations.

TABLE II

\begin{tabular}{c|c|c|c|c|c|c}
\hline \hline Molecules & $\sigma_{1} / \sigma_{0}$ & $\sigma_{2} / \sigma_{0}$ & $\sigma_{3} / \sigma_{0}$ & $\begin{array}{c}\text { Long } \\
\text { axis }\end{array}$ & $\begin{array}{c}\text { Short } \\
\text { axis }\end{array}$ & $\begin{array}{c}C_{2} \\
\text { axis }\end{array}$ \\
\hline wedge-like & 0.2 & 0.5 & -0.4 & $\boldsymbol{n}$ & $\boldsymbol{l}$ & $\boldsymbol{n}$ \\
banana-like & 0.2 & -0.4 & 0.5 & $\boldsymbol{l}$ & $\boldsymbol{n}$ & $\boldsymbol{n}$
\end{tabular}

\subsection{Wedge-shaped biaxial molecules}

In the system of wedge-shaped biaxial molecules, on decreasing the temperature we meet the first order transition from the isotropic to the uniaxial nematic phase at $T_{\mathrm{IN}}=0.618$ and the second order transition to the biaxial 
nematic phase at $T_{\mathrm{NB}}=0.401$. The temperature dependence of the order tensors is presented in Fig. 1. The temperature dependence of the elastic constants and the FE coefficients are presented in Figs. 2 and 3, respectively.

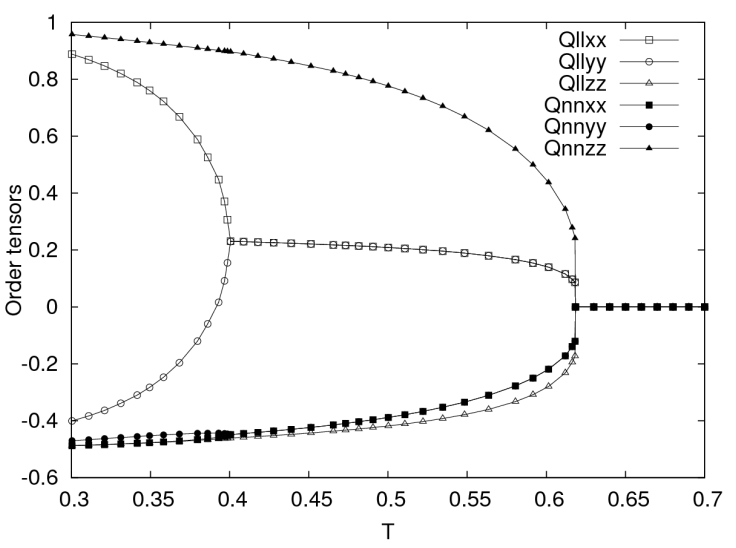

Fig. 1. The temperature dependence of the order tensors for wedge-shaped biaxial molecules.

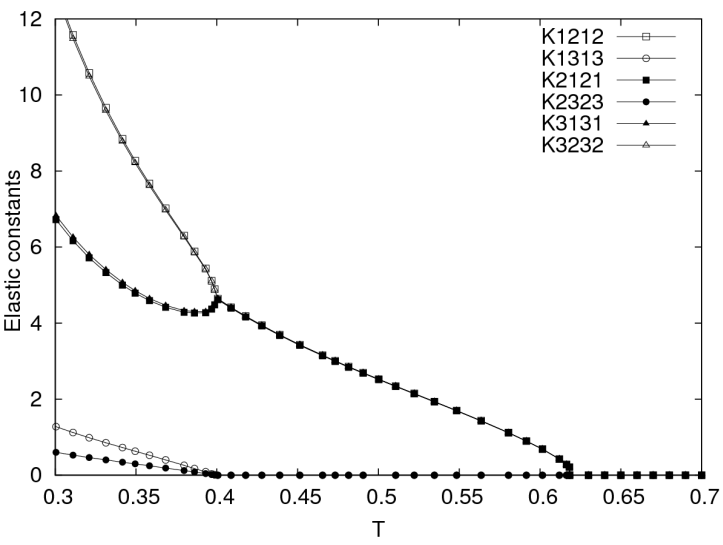

Fig. 2. The temperature dependence of the elastic constants for wedge-shaped biaxial molecules. The squares, triangles, and circles denote deformations with $\boldsymbol{N}$ splay, $\boldsymbol{N}$ bend, and $\boldsymbol{N}$ constant, respectively. The empty (filled) symbols indicate the larger (smaller) parameter.

The values of $Q_{z z}^{n n}$ show that long molecule axes are directed along the $Z$ axis in the whole nematic region, whereas the values of $Q_{x x}^{l l}$ reveal the alignment of short molecule axes along the $X$ axis and it is enhanced in the biaxial nematic phase. On the other hand, the values of $Q_{y y}^{l l}$ decrease after the transition from the uniaxial nematic to the biaxial nematic phase and change the sign from positive to negative. The splay elastic constant $K_{1}$ splits into $K_{1212}$ and $K_{2121}$. The bend elastic constant $K_{3}$ splits into $K_{3232}$ and $K_{3131}$. Let us note that the equality $K_{1}=K_{3}$ is accidental and results from neglecting order parameters $F_{\mu \nu}^{(j)}$ with $j>2$. The splay FE coefficient $e_{1}$ splits into $a_{4}$ and $a_{6}\left(a_{4}>a_{6}>0\right)$. The bend

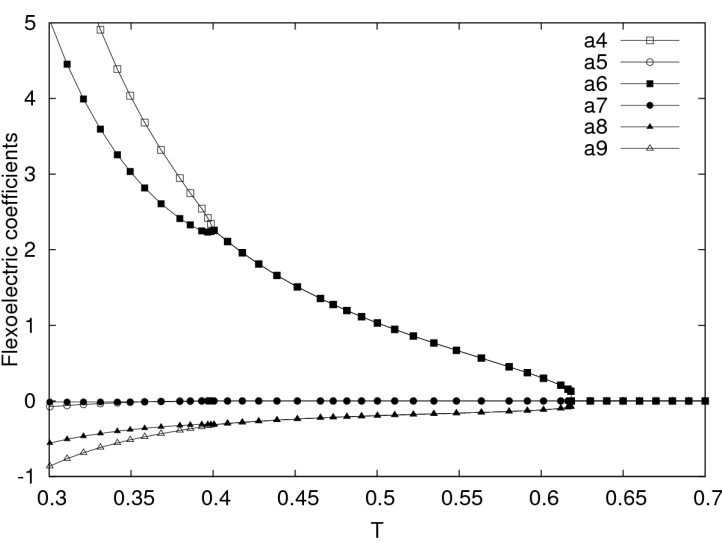

Fig. 3. The temperature dependence of the flexoelectric coefficients for wedge-shaped biaxial molecules. Symbols have the same meaning as in Fig. 2.

FE coefficient $-e_{3}$ splits into $a_{8}$ and $a_{9}\left(0>a_{8}>a_{9}\right)$. The coefficients $a_{5}$ and $a_{7}$ are small and almost always negative.

\subsection{Banana-shaped biaxial molecules}

In the system of banana-shaped biaxial molecules, on decreasing the temperature we meet the first order transition from the isotropic to the uniaxial nematic phase at $T_{\mathrm{IN}}=0.595$ and the second order transition to the biaxial nematic phase at $T_{\mathrm{NB}}=0.382$. The temperature dependence of the order tensors is presented in Fig. 4. The temperature dependence of the elastic constants and the FE coefficients are presented in Figs. 5 and 6 , respectively.

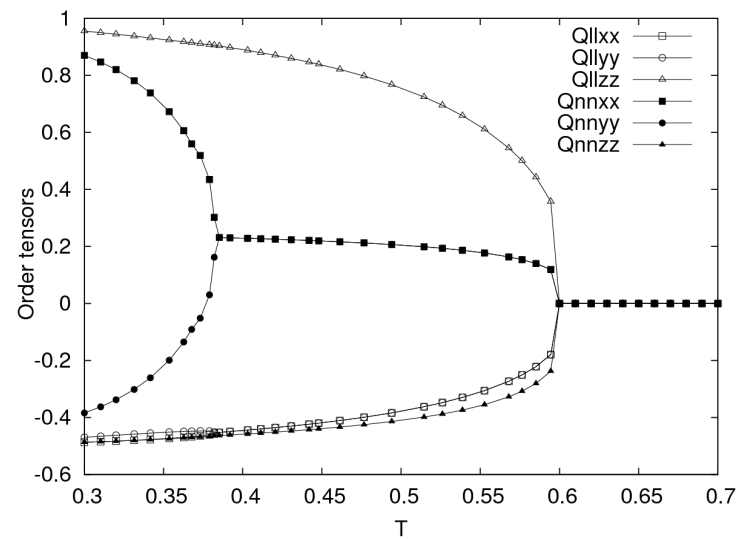

Fig. 4. The temperature dependence of the order tensors for banana-shaped biaxial molecules.

According to the values of $Q_{z z}^{l l}$ long molecule axes are directed along the $Z$ axis in the whole nematic region, whereas the values of $Q_{x x}^{n n}$ show the alignment of short molecule axes along the $X$ axis and it is enhanced in the biaxial nematic phase. The values of $Q_{y y}^{n n}$ decrease and change the sign from positive to negative near the transition from the uniaxial nematic to the biaxial nematic 


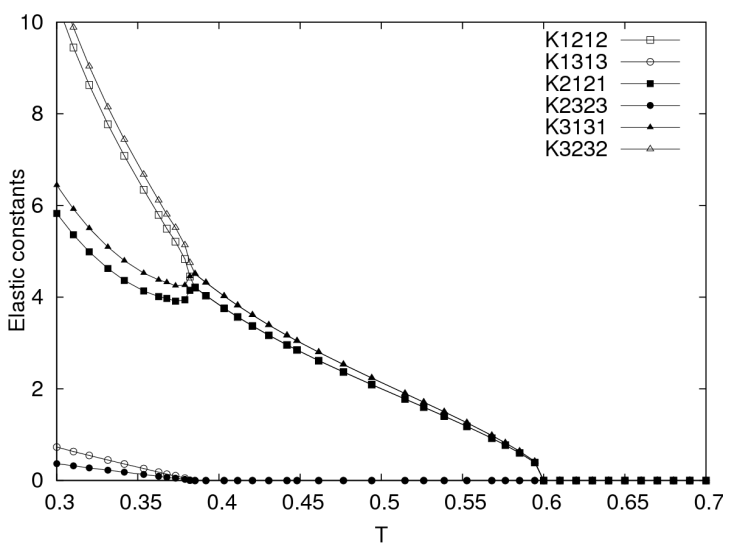

Fig. 5. The temperature dependence of the elastic constants for banana-shaped biaxial molecules. Symbols have the same meaning as in Fig. 2.

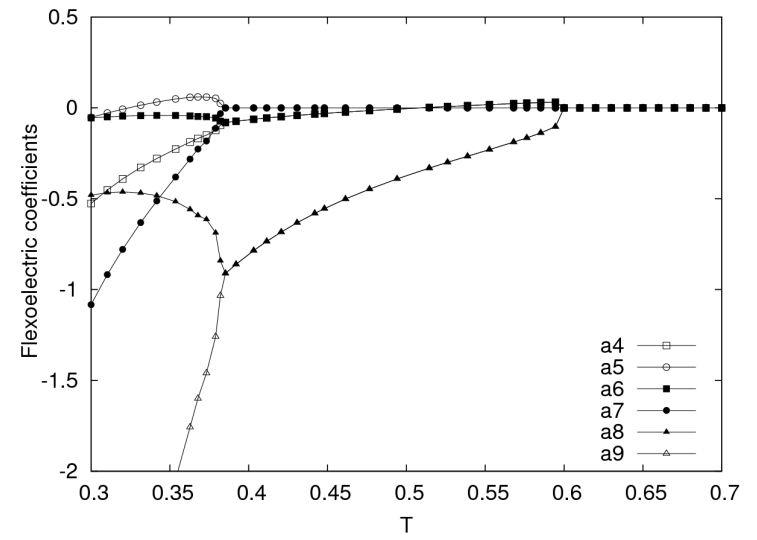

Fig. 6. The temperature dependence of the flexoelectric coefficients for banana-shaped biaxial molecules. Symbols have the same meaning as in Fig. 2.

phase. The behaviour of the elastic constants is similar to the case of the wedge-shaped molecules because the FE term is small in both cases. The bend FE coefficient $-e_{3}$ splits into $a_{8}$ and $a_{9}\left(0>a_{8}>a_{9}\right)$. The splay FE coefficient $e_{1}$ is smaller than $e_{3}$ and it splits into $a_{4}$ and $a_{6}$. The coefficients $a_{5}$ and $a_{7}$ are again small but comparable with $a_{4}$ and $a_{6}$. The sign of some coefficients can change on changing the temperature.

\section{Conclusions}

In this paper, the statistical theory was used to study the temperature dependence of the order parameters, elastic constants, and FE coefficients of biaxial nematic liquid crystals. In order to calculate these macroscopic parameters one needs the one-particle distribution function and the potential energy of molecular interactions. The two physical systems were considered. The splittings of the FE coefficients and the elastic constants were obtained at the uniaxial-biaxial nematic transition. New small FE coefficients appeared at the transition. The ordering of the split FE coefficients corresponds to the ordering of the split elastic constants.

The FE coefficients were proportional to the dipole moment component parallel to the molecule $C_{2 v}$ symmetry axis. This was the result of the interactions potential symmetry. The beaviour of the main FE coefficients, $e_{1}$ for the wegde-shaped molecules and $e_{3}$ for the banana-shaped molecules, is clear and it is in agreement with previous studies [28]. On the other hand, it seems that other FE coefficients should be interpreted with caution. It is possible that higher order parameters can have a significant contribution.

At present stage, the direct comparison between the theory and the experiment in not possible because to our knowledge the FE coefficients have not been measured for the biaxial nematic phase. What is more, even for the uniaxial nematic phase the experimental data are still scarce and sometimes contradictory [29]. However, when biaxial nematic phases become more widespread, the presented theory will be helpful in practical applications.

In the case of the uniaxial nematic phase, the FE coefficients can be measured using hybrid-aligned nematic [30] or twisted nematic (TN) [31, 32] devices with in-plane switching electric fields. Recently, Outram and Elston [33] employed a crystal rotation method to measure the difference $e_{1}-e_{3}$ in a TN cell. Values of $e_{1}-e_{3}$ for $\mathrm{E} 7$ were measured to be $7.8 \pm 1.0 \mathrm{pC} / \mathrm{m}$ at $298 \mathrm{~K}$. E7 is a four-component mixture composed of cyano-based calamitic molecules (5CB, 7CB, 80CB, 5CT). The elastic constants were $K_{1}=10.7 \mathrm{pN}, K_{2}=6.5 \mathrm{pN}$, and $K_{3}=16 \mathrm{pN}$. The experimental nematic-isotropic phase transition is $331 \mathrm{~K}$. Molecular dynamics simulations of E7 were reported by Pelaez and Wilson [34]. We would like to give estimates from our theory assuming reasonable values of the model parameters. If we assume the molecular length $\sigma_{0}=10 \AA$, the interaction energy $\epsilon=0.1 \mathrm{eV}$, and the electric dipole $\mu=3 \mathrm{D}$ (for $5 \mathrm{CB}$ alone it is $4.85 \mathrm{D}$ ), then we can estimate the values of the FE coefficients $\mu / \sigma_{0}^{2}=10 \mathrm{pC} / \mathrm{m}$, the elastic constants $\epsilon / \sigma_{0}=16 \mathrm{pN}$, and the temperature scale $\epsilon / k_{\mathrm{B}}=1160 \mathrm{~K}$.

\section{Acknowledgments}

The author is grateful to J. Spałek for his support.

\section{References}

[1] M.J. Freiser, Phys. Rev. Lett. 24, 1041 (1970).

[2] L.J. Yu, A. Saupe, Phys. Rev. Lett. 45, 1000 (1980).

[3] K. Severing, K. Saalwachter, Phys. Rev. Lett. 92, 125501 (2004).

[4] L.A. Madsen, T.J. Dingemans, M. Nakata, E.T. Samulski, Phys. Rev. Lett. 92, 145505 (2004).

[5] B.R. Acharya, A. Primak, S. Kumar, Phys. Rev. Lett. 92, 145506 (2004).

[6] K. Merkel, A. Kocot, J.K. Vij, R. Korlacki, G.H. Mehl, T. Meyer, Phys. Rev. Lett. 93, 237801 (2004). 
[7] L. Longa, P. Grzybowski, S. Romano, E. Virga, Phys. Rev. E 71, 051714 (2005).

[8] R. Berardi, L. Muccioli, S. Orlandi, M. Ricci, C. Zannoni, J. Phys., Condens. Matter 20, 463101 (2008).

[9] L. Longa, G. Pająk, T. Wydro, Phys. Rev. E 76, 011703 (2007).

[10] R.B. Meyer, Phys. Rev. Lett. 22, 918 (1969).

[11] J. Prost, J.P. Marcerou, J. Phys. (Paris) 38, 315 (1977).

[12] A. Kapanowski, Phys. Rev. E 77, 052702 (2008).

[13] C. Denniston, J.M. Yeomans, Phys. Rev. Lett. 87, 275505 (2001).

[14] A.J. Davidson, N.J. Mottram, Phys. Rev. E 65, 051710 (2002).

[15] L.A. Parry-Jones, R.B. Meyer, S.J. Elston, J. Appl. Phys. 106, 014510 (2009).

[16] J.S. Patel, R.B. Meyer, Phys. Rev. Lett. 58, 1538 (1987).

[17] A.E. Blatch, M.J. Coles, B. Musgrave, H.J. Coles, Mol. Cryst. Liq. Cryst. 401, 161 (2003).

[18] F. Castles, S.M. Morris, H.J. Coles, Phys. Rev. E 80, 031709 (2009).

[19] M. Cepic, B. Zeks, Phys. Rev. Lett. 87, 085501 (2001).

[20] F. Castles, S.M. Morris, E.M. Terentjev, H.J. Coles, Phys. Rev. Lett. 104, 157801 (2010).

[21] G. Barbero, I. Dozov, J.F. Palierne, G. Durand, Phys. Rev. Lett. 56, 2056 (1986).
[22] T. Porenta, M. Ravnik, S. Zumer, Soft Matter 7, 132 (2011).

[23] A. Kapanowski, Phys. Rev. E 55, 7090 (1997).

[24] M. Fiałkowski, A. Kapanowski, K. Sokalski, Mol. Cryst. Liq. Cryst. 265, 371 (1995).

[25] R. Rosso, Liq. Cryst. 34, 737 (2007).

[26] P.J. Camp, M.P. Allen, J. Chem. Phys. 106, 6681 (1997).

[27] S. Stallinga, G. Vertogen, Phys. Rev. E 49, 1483 (1994).

[28] A. Kapanowski, Opto-Electron. Rev. 16, 9 (2008).

[29] A.G. Petrov, in: Physical Properties of Liquid Crystals: Nematics, Eds. D. Dunmur, A. Fukuda, G.L. Luckhurst, INSPEC, The Institution of Electrical Engineers, London 2001.

[30] I. Dozov, P. Martinot-Lagarde, G. Durand, J. Phys. Lett. 43, 365 (1982).

[31] R.A. Ewings, C. Kischka, L.A. Parry-Jones, S.J. Elston, Phys. Rev. E 73, 011713 (2006).

[32] E.K. Tidey, L.A. Parry-Jones, S.J. Elston, Liq. Cryst. 34, 251 (2007).

[33] B.I. Outram, S.J. Elston, Liq. Cryst. 39, 149 (2012).

[34] J. Pelaez, M. Wilson, Phys. Chem. Chem. Phys. 9, 2968 (2007). 